\title{
Treatment of Acid Orange 24 Solutions with Dimensionally Stable Anodes
}

\author{
M.T. León, * G.G. Pomposo, G.J. Suárez, S.S. Vega \\ Facultad de Ingeniería, Benemérita Universidad Autónoma de Puebla \\ Edificio 123, Cd. Universitaria, Puebla, Pue, C.P. 72570, MEXICO
}

Received 25 April 2008; accepted 18 October 2008

\begin{abstract}
Removal of acid orange 24 color was studied in an electrochemical cell composed of a dimensional stable anode (DSA), a stainless steel cathode and an electrolyte media containing sodium sulfate, sodium chloride, and two commercial surfactants named Uniperol y Leophen, corresponding to wetting and dispersant agents, both of them required to favor the color incorporation inside the fiber. DSA used in this study comprises a titanium mesh covered by a layer of iridium, and a stainless steel plate covered by a layer of iridium-tin mixture.

Experimental approach allowed getting an inside of the rol played by sulfate, chloride and surfactants for both color and chemical oxygen demand (COD) removal. Obtained results have shown that the Ir-Sn electrode provides the highest color removal under chloride mediated oxidant conditions.

Evaluation of the DSA performance in solutions containing surfactants has shown that obtained color removal is similar for both electrodes; otherwise the COD removal attained with the Ir DSA is about six times the one obtained with the Ir-Sn electrode.
\end{abstract}

Keywords: azo color, DSA, textile wastewater, electrochemical treatment.

\section{Introducción}

Los colorantes de tipo ácido han sido usados en el teñido de fibras proteínicas (lana, seda) y algunas de tipo poliamídicas (nylon). Una característica de los colorantes ácidos es la presencia de grupos sulfito $\left(\mathrm{Na}_{2} \mathrm{SO}_{3}\right)$ lo cual les confiere solubilidad y una carga negativa. Asimismo, en los colorantes amarillos, naranjas y rojos está presente el grupo azo $(\mathrm{N}=\mathrm{N})$; mientras que en el grupo de azules y verdes existe un grupo carbonilo. El grado de afinidad de los colorantes con las fibras va de moderado a fuerte; en el primer caso es posible que en la fibra ocurra una migración del color y se alcance un teñido uniforme, pero con una moderada

\footnotetext{
* Corresponding author. E-mail address: teutli23@hotmail.com
} 
resistencia al deslavado; por el contrario, una afinidad fuerte evita la migración, pero favorece que el color tenga mayor resistencia al lavado; un teñido uniforme con alta resistencia al deslavado se puede obtener haciendo uso de surfactantes, los cuales incrementan la solubilidad del colorante y su afinidad con la fibra [1].

En general, un agua residual de procesos de teñido y acabado textil puede contener una gama amplia de contaminantes que van desde impurezas de las fibras, hasta los compuestos químicos agregados durante el procesamiento para obtener hilos, tejidos, etc. Por esta razón, un vertido de agua residual puede contener una extensa variabilidad en cuanto a $\mathrm{pH}$, contenido de color, Demanda Química de Oxígeno (DQO) y Demanda Bioquímica de Oxígeno (DBO) [2]. En adición a lo anterior, un vertido de colorantes ácidos puede representar una amenaza al medio ambiente por su contenido de cloruro de sodio, ya que por fotoactivación cualquiera de los subproductos clorados $\left(\mathrm{Cl}, \mathrm{OCl}^{-}, \mathrm{HOCl}^{-}\right)$puede llegar a reaccionar con los compuestos orgánicos propiciando la formación de subproductos organoclorados, los cuales ejercerían mayor toxicidad que las moléculas originales.

El tratamiento de aguas residuales textiles por métodos físico-químicos, como se realiza en las plantas de tratamiento de agua residual (PTAR), logra incidir de manera importante en el contenido orgánico y de manera mínima en el color, además de que se tiene el inconveniente del volumen de lodos que se genera. Como un tratamiento alternativo para la remoción de color, se han explorado métodos electroquímicos como la electrocoagulación y la mineralización, con los cuales la remoción de colorantes ácidos ha tenido éxito [3, 4]; en la electrocoagulación, una ventaja adicional es que se puede tener un proceso lateral de flotación, lo que ayuda en la remoción del color [5]. Sin embargo, uno de los inconvenientes es que eventualmente el problema no ha sido resuelto, sino que se traslada a otra esfera: la de los desechos sólidos.

Recientemente, el uso de ánodos dimensionalmente estables (DSA por sus siglas en inglés) ha mostrado éxito en generar condiciones fuertemente oxidantes; la actividad oxidante en estos electrodos es función del material con el que fueron construidos, así pueden catalogarse como activos $\mathrm{Pt}, \mathrm{IrO}_{2}$, acero inoxidable, mientras que el $\mathrm{PbO}_{2}$ y el $\mathrm{SnO}_{2}$ se consideran como no-activos. Ejemplos de aplicación de una gama de electrodos en la degradación de colorantes ha sido informada por Rajkumar [6] y Hastie [7]. Estos trabajos consideran variaciones en la composición química del medio, así como un seguimiento de la evolución de subproductos por medio de análisis de cromatografía de líquidos (HPLC), carbón orgánico total (COT) y DQO.

Respecto a los posibles mecanismos de oxidación de compuestos orgánicos mediante DSA, se tiene el trabajo de Savall [8], quien reportó que el proceso de oxidación sobre los DSA lleva la superficie a un estado de oxidación más alta $\left(\mathrm{MO}_{\mathrm{x}+1}\right)$ el cual puede devenir en 2 mecanismos: 1) M1 donde se propicia la reacción de $\mathrm{MO}_{\mathrm{x}+1}$ con agua para formar radicales hidroxilo que inicialmente son adsorbidos en la superficie del electrodo, para después oxidar el sustrato objetivo; 2) $\mathrm{M} 2$ donde el $\mathrm{MO}_{\mathrm{x}+1}$ oxida el sustrato directamente, llevando el óxido metálico a un estado de oxidación menor. A este respecto, Comninellis [9] ha indicado que es más probable que el mecanismo M1 ocurra en los DSA basados 
en Sn, mientras que el M2 tenga lugar en los DSA basados en Ir. Un informe de Rodgers [10] describe la oxidación de fenoles clorados utilizando un DSA de Ir y otro de Sn; en sus resultados los autores observaron que la electrólisis fue más eficiente en el ánodo de Sn, aunque el de Ir fue más resistente al envenenamiento.

Entre los trabajos de investigación enfocados a obtener información adicional acerca de la influencia del cloruro de sodio en la decoloración y reducción de la DQO, cuando se trata el efluente con colorantes azo (ácidos) se encuentran los trabajos de Sanjay et al. [11] y de Kupferle et al. [12]. En el primero, se menciona el tratamiento de un efluente textil con una celda de flujo teniendo como electrodos un DSA y acero inoxidable. El efluente referido estaba compuesto por una mezcla de colorantes azo y un alto contenido de cloruros; en sus conclusiones se menciona el hecho de que, aplicando densidades de corriente entre 10 y $40 \mathrm{~mA} \mathrm{~cm}^{-2}$ y manteniendo el $\mathrm{pH}$ muy cercano a la neutralidad ( $\mathrm{pH}=7$ 8), se logra una importante decoloración y reducción en DQO, debido a la oxidación de las moléculas de colorante por acción de mediadores tales como $\mathrm{HOCl}^{-}$y $\mathrm{OCl}^{-}$, radicales que se vuelven inestables a $\mathrm{pH}$ ácidos o alcalinos; esto último se confirmó con experimentos a pH extremos, donde la eliminación de DQO fue mucho menor. Asimismo, ellos presentan análisis IR que confirmar la ausencia de productos secundarios de tipo organoclorado.

En el trabajo de Kupferle et al. [12] se analiza el tratamiento de aguas residuales textiles conteniendo colorantes azo en un medio electrolítico que incluye $\mathrm{NaCl}$ y $\mathrm{Na}_{2} \mathrm{SO}_{4}$. Los experimentos fueron realizados en una celda electrolítica conformada por un ánodo de grafito y un cátodo de acero inoxidable, la composición del electrólito se fijó en $0.05 \mathrm{~N}$, y se consideran variaciones desde $0.05 \mathrm{~N}$ de $\mathrm{NaCl}: 0 \mathrm{~N}$ de $\mathrm{Na}_{2} \mathrm{SO}_{4}$ hasta $0 \mathrm{~N}$ de $\mathrm{NaCl}: 0.05 \mathrm{~N}$ de $\mathrm{Na}_{2} \mathrm{SO}_{4}$. Aplicando una densidad de corriente de $5 \mathrm{~mA} \mathrm{~cm}^{-2}$, en sus resultados, observaron que la decoloración por medio de $\mathrm{NaCl}$ (oxidación indirecta) es mayor en un orden de magnitud que la lograda con $\mathrm{Na}_{2} \mathrm{SO}_{4}$ (oxidación directa). De sus mediciones experimentales muestran un gráfico de constantes de primer orden versus concentración de cloruros, en el cual se definen claramente 3 regiones: a) la limitada por la producción de cloro oxidado $(0-0.01 \mathrm{~N} \mathrm{NaCl})$, b) la limitada por la oxidación química del colorante $(0.01 \mathrm{~N}-0.04 \mathrm{~N} \mathrm{NaCl})$; y c) la limitada por la transferencia de electrones $(>0.04 \mathrm{~N} \mathrm{NaCl})$.

Considerando que la receta de un baño típico de colorantes ácidos, con enlaces azo, incluye no sólo $\mathrm{NaCl}_{\text {y }} \mathrm{Na}_{2} \mathrm{SO}_{4}$, sino que para tener un teñido homogéneo y resistente al lavado se deben incluir surfactantes que permitan la dispersión (igualante) y la adherencia (humectante) del colorante en la fibra, es necesario valorar la presencia de los surfactantes en el tratamiento electrolítico de aguas residuales textiles conteniendo colorantes azo.

En este trabajo se presenta la degradación de disoluciones típicas del colorante naranja ácido 24, mediante DSA de Ir e Ir-Sn, considerando valorar la remoción de color y DQO en presencia de un electrólito primario $\left(\mathrm{Na}_{2} \mathrm{SO}_{4}\right)$, que permitirá la oxidación directa, un electrólito secundario $(\mathrm{NaCl})$ que producirá los mediadores para la oxidación indirecta y los surfactantes. 


\section{Experimental \\ Reactivos}

Colorante naranja ácido 24 (CIBA), con la estructura molecular que se presenta en la Fig. 1; surfactantes comerciales Uniperol (Humectante $=\mathrm{H}$ ) y Leophen (Igualante=I); sales grado reactivo de $\mathrm{NaCl}, \mathrm{Na}_{2} \mathrm{SO}_{4}$ marca Baker, y tubos $\mathrm{HACH}$ para determinación espectrofotométrica de DQO.

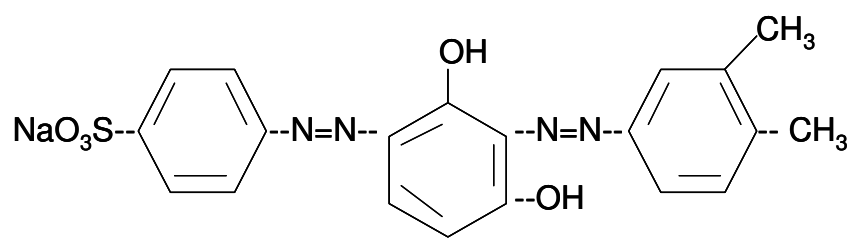

Figura 1. Estructura molecular del colorante naranja ácido 24, en la que se observa el grupo sulfito $\left(\mathrm{NaSO}_{3}\right)$ que le da el carácter ácido y el grupo azo $(\mathrm{N}=\mathrm{N})$ que le confiere el color naranja.

\section{Aparatos}

Se utilizó una fuente de tensión marca PROTEK modelo 3003B para imponer una corriente contínua; un conductímetro marca CONDUCTRONIC, modelo CL8; un pHmetro portátil marca CONDUCTRONIC, modelo pH10; un espectrofotómetro HACH, modelo DR2500.

\section{Celda experimental}

Para la celda se utilizó un vaso de precipitados de $200 \mathrm{~mL}$, en el que se colocaron $100 \mathrm{~mL}$ de disolución y se sumergieron los electrodos. El cátodo fue una placa de acero inoxidable 303 , de $5 \times 1 \mathrm{~cm}^{2}$. Los ánodos DSA fueron provistos por la compañía ELTECH; el primero consta de una placa recubierta de Ir-Sn y el segundo es una malla de Ti recubierta con una película de $\mathrm{Ir}$, ambos de $5 \times 1 \mathrm{~cm}$. Los electrodos se colocaron verticalmente, de forma paralela, con una distancia entre ellos de $1 \mathrm{~cm}$.

\section{Método experimental}

La preparación de las disoluciones se realizó de acuerdo con las instrucciones del proveedor químico, quien indicó las concentraciones probables de color y surfactantes residuales de un baño de teñido. De acuerdo a la información obtenida: $250-500 \mathrm{mg} \mathrm{L}^{-1}$ de colorante naranja ácido $24 ; 1-2 \mathrm{ml} \mathrm{L}^{-1}$ de $\mathrm{H}$ e I; la concentración del electrólito primario $\mathrm{Na}_{2} \mathrm{SO}_{4} 10 \mathrm{~g} \mathrm{~L} \mathrm{~L}^{-1}$, y la del electrólito secundario $\mathrm{NaCl} 5 \mathrm{~g} \mathrm{~L}^{-1}$; el sulfato es parte esencial de un baño de teñido de colorantes ácidos; el cloruro, aunque en menor cantidad, es un componente que generará los compuestos oxidantes mediadores en la degradación del color. Esto último determinó que se valorase la acción de cada uno de los electrodos considerando como referencia el baño de teñido con el electrólito primario y por etapas, incluir el electrólito secundario y los surfactantes. La densidad de corriente aplicada fue de $5 \mathrm{~mA} \mathrm{~cm}^{-2}$, lo cual corresponde a un valor donde los productos de electrólisis no modifican sustancialmente el $\mathrm{pH}$ logrando mantener 
la disolución en valores cercanos a la neutralidad. Las combinaciones experimentales consideradas se indican en la Tabla 1.

Tabla 1. Combinaciones experimentales consideradas.

\begin{tabular}{|c|c|c|c|c|c|c|}
\hline Exp & $\begin{array}{c}\text { Color } \\
\mathrm{ppm}\end{array}$ & $\begin{array}{c}\mathrm{Na}_{2} \mathrm{SO}_{4} \\
\mathrm{~g} \mathrm{~L}^{-1}\end{array}$ & $\begin{array}{c}\mathrm{NaCl} \\
\mathrm{g} \mathrm{L}^{-1}\end{array}$ & $\begin{array}{c}\mathrm{H} \\
\mathrm{ml} \mathrm{L}^{-1}\end{array}$ & $\begin{array}{c}\mathrm{I} \\
\mathrm{ml} \mathrm{L}^{-1}\end{array}$ & $\begin{array}{c}\text { Anodo } \\
\mathrm{DSA}\end{array}$ \\
\hline 1 & 350 & 10 & 0 & 0 & 0 & $\mathrm{Ir}$ \\
\hline 2 & 350 & 10 & 5 & 0 & 0 & $\mathrm{Ir}$ \\
\hline 3 & 350 & 10 & 0 & 1 & 1 & $\mathrm{Ir}$ \\
\hline 4 & 350 & 10 & 5 & 1 & 1 & $\mathrm{Ir}$ \\
\hline 5 & 350 & 10 & 0 & 0 & 0 & $\mathrm{Ir}-\mathrm{Sn}$ \\
\hline 6 & 350 & 10 & 5 & 0 & 0 & $\mathrm{Ir}-\mathrm{Sn}$ \\
\hline 7 & 350 & 10 & 0 & 1 & 1 & $\mathrm{Ir}-\mathrm{Sn}$ \\
\hline 8 & 350 & 10 & 5 & 1 & 1 & Ir-Sn \\
\hline
\end{tabular}

\section{Resultados y discusión}

A continuación se discuten los resultados obtenidos en remoción de color y DQO. Sobre la base de las definiciones de Kupferle [12], se considera que el mecanismo de oxidación directa corresponde al electrólito conteniendo $\mathrm{Na}_{2} \mathrm{SO}_{4}$, mientras que el mecanismo de oxidación indirecta corresponde al electrólito de $\mathrm{Na}_{2} \mathrm{SO}_{4}+\mathrm{NaCl}$.

La Fig. 2 muestra la remoción de color al utilizar como ánodo el electrodo de IrSn. Se observa que en el mecanismo de oxidación directa, la máxima remoción se alcanza en 150 min y corresponde a $170 \mathrm{ppm}$, mientras que en el mecanismo de oxidación indirecta, la presencia de cloruros favorece que la remoción se incremente en un $90 \%$, alcanzando a remover 325 ppm.

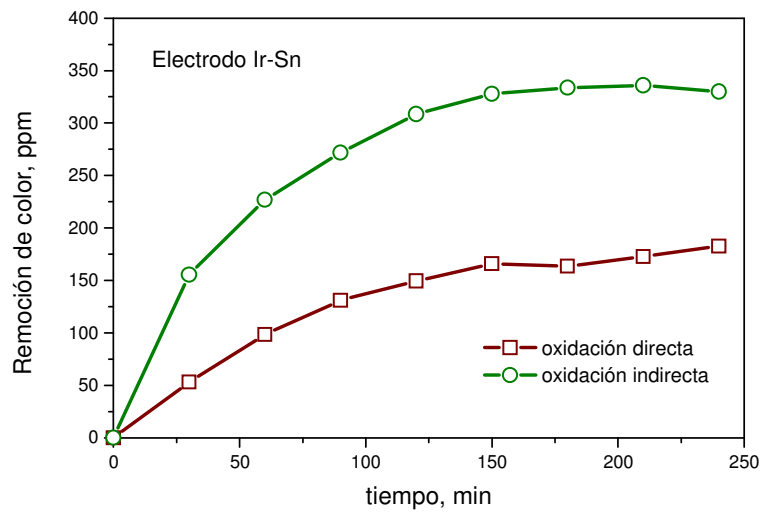

Figura 2. Remoción de color obtenida con el ánodo de $\mathrm{Ir}-\mathrm{Sn}$ cuando el electrólito corresponde a condiciones de oxidación directa $\left(\mathrm{Na}_{2} \mathrm{SO}_{4}\right)$ y oxidación indirecta $\left(\mathrm{Na}_{2} \mathrm{SO}_{4}+\mathrm{NaCl}\right)$.

La modificación del electrólito al incluir los surfactantes ( $\mathrm{H}$ e I) afecta la capacidad de remoción de color. En la Fig. 3 puede observarse que existe una demora de 30 minutos en la respuesta de remoción de color; asimismo, en los 
siguientes 90 minutos existe una diferencia mínima entre la oxidación directa y la indirecta, diferencia que desaparece después de los 120 minutos. Para comparar con el proceso de oxidación indirecta, se toma como referencia el tiempo de 150 minutos, que corresponde a la máxima remoción en ausencia de surfactantes, observándose que la inclusión de los últimos interfiere con el proceso, ya que la remoción alcanzada es de 190 ppm, lo cual es aproximadamente el $60 \%$ de la obtenida en ausencia de surfactantes.

En la Fig. 4 se muestra la remoción de color utilizando el ánodo de Ir. Como puede observarse, el proceso de oxidación directa presenta un período de activación de 30 minutos, el cual está ausente en el proceso de oxidación indirecta. En un tiempo de 4 horas, la remoción obtenida por oxidación directa es de $150 \mathrm{ppm}$, mientras que por oxidación indirecta se obtiene una remoción de 225 ppm. Estos resultados son menores que los obtenidos con el electrodo de IrSn. Adicionalmente, el proceso con el electrodo de Ir resulta más lento, ya que a las 4 horas la tendencia de los datos indica que el sistema no ha alcanzado el máximo de remoción.

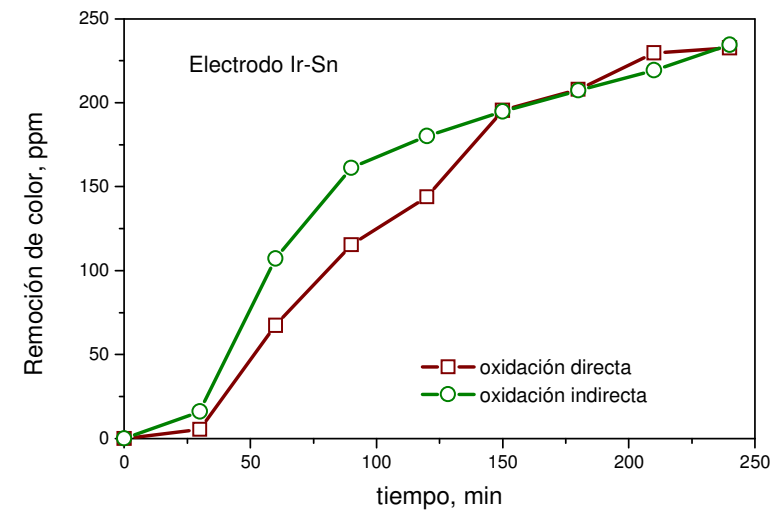

Figura 3. Remoción de color obtenida con el ánodo de $\mathrm{Ir}-\mathrm{Sn}$ cuando el electrólito corresponde a condiciones de oxidación directa $\left(\mathrm{Na}_{2} \mathrm{SO}_{4}\right)$ y oxidación indirecta $\left(\mathrm{Na}_{2} \mathrm{SO}_{4}+\mathrm{NaCl}\right)$ en presencia de surfactantes (Humectante e Igualante).

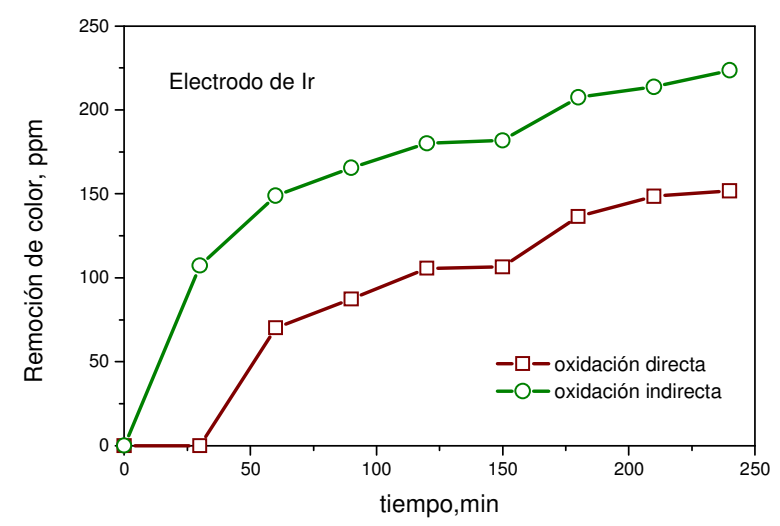

Figura 4. Remoción de color obtenida con el ánodo de Ir cuando el electrólito corresponde a condiciones de oxidación directa $\left(\mathrm{Na}_{2} \mathrm{SO}_{4}\right)$ y oxidación indirecta $\left(\mathrm{Na}_{2} \mathrm{SO}_{4}+\mathrm{NaCl}\right)$. 
Los resultados del tratamiento con el electrodo de Ir en presencia de surfactantes se presentan en la Fig. 5, observándose que la respuesta de remoción se modifica, siendo prácticamente la misma en oxidación directa que en la indirecta durante los primeros 60 minutos. Para la comparación con los valores alcanzados en el medio sin surfactantes se toma como referencia 4 horas. Se observa que la remoción alcanzada en la oxidación directa es $30 \mathrm{ppm}$ mayor que en ausencia de surfactantes y que en el mecanismo de oxidación indirecta se obtienen valores similares a los obtenidos sin surfactantes.

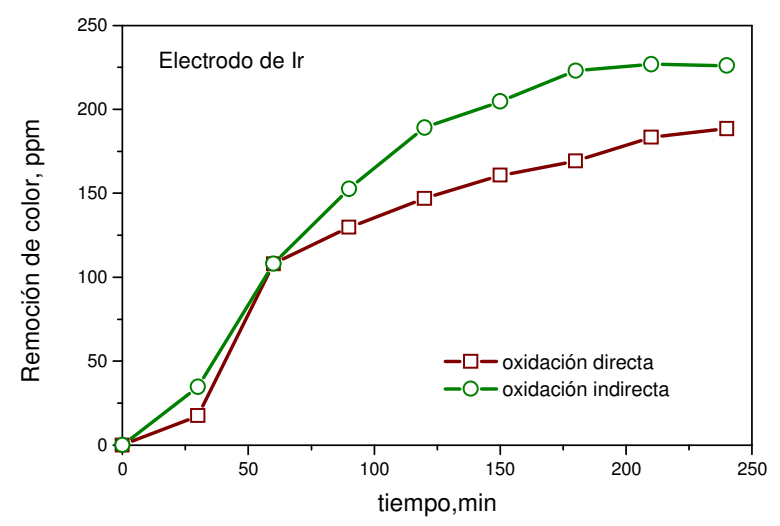

Figura 5. Remoción de color obtenida con el ánodo de Ir cuando el electrólito corresponde a condiciones de oxidación directa $\left(\mathrm{Na}_{2} \mathrm{SO}_{4}\right)$ y oxidación indirecta $\left(\mathrm{Na}_{2} \mathrm{SO}_{4}+\mathrm{NaCl}\right)$ en presencia de surfactantes (Humectante e Igualante).

Es importante puntualizar que la inclusión de los surfactantes en la disolución de color hace que la DQO inicial se incremente de manera importante, ya que la disolución de color y electrólito registró una DQO de 500 ppm, mientras que la disolución preparada con color, electrólito y surfactantes registró una DQO de 6000 ppm, lo cual corresponde a 12 veces la DQO del sistema sin surfactantes.

En la Fig. 6 se muestran los resultados de remoción de DQO para ambos electrodos. Como puede observarse, para el electrodo de Ir-Sn la oxidación directa requiere un período de activación de 30 minutos, mientras que en la oxidación indirecta, en 60 minutos se alcanzan a remover 310 ppm de DQO y el máximo corresponde a 580 ppm en un tiempo de 2 horas; después de ese tiempo la respuesta es aleatoria.

En el caso de la remoción lograda con el electrodo de Ir, es evidente que el rendimiento de remoción de este electrodo es superior al observado con el de IrSn, ya que por oxidación directa, en 30 minutos, se alcanza a remover 1100 ppm de DQO lo cual es 1.5 veces mayor que la máxima cantidad removida con el electrodo de Ir-Sn. En cuanto a la oxidación indirecta, el máximo de remoción se alcanza en 60 minutos y es 6 veces el valor máximo obtenido con el electrodo de Ir-Sn. 


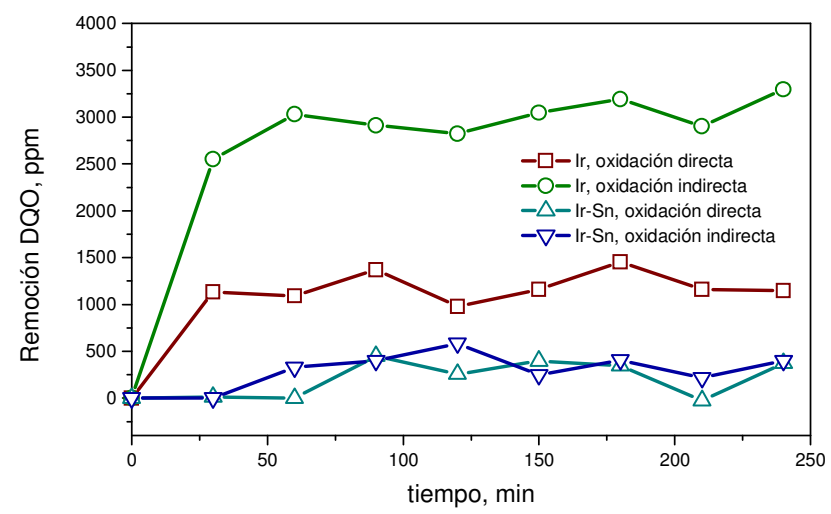

Figura 6. Remoción de Demanda Química de Oxígeno (DQO) obtenidas con los ánodos de Ir e Ir-Sn cuando el electrólito corresponde a condiciones de oxidación directa $\left(\mathrm{Na}_{2} \mathrm{SO}_{4}\right)$ y oxidación indirecta $\left(\mathrm{Na}_{2} \mathrm{SO}_{4}+\mathrm{NaCl}\right)$ en presencia de surfactantes.

\section{Conclusiones}

A partir de los resultados presentados se puede concluir que el uso del electrodo de Ir-Sn presenta un rendimiento mayor en la remoción de color en ausencia de surfactantes, mientras que el de Ir da una mejor respuesta en la remoción de DQO en presencia de surfactantes.

Los surfactantes afectan el desempeño de los DSA en cuanto a la remoción de color, ya que los resultados son prácticamente similares para ambos electrodos. Por el contrario, el análisis de DQO permite establecer diferencias entre ellos ya que, en presencia de surfactantes, los cloruros y sus posibles mediadores permiten que el mecanismo de oxidación indirecta logre una remoción importante de la DQO, la cual, a tiempos similares, llega a ser de hasta 6 veces la que se logra con el electrodo de Ir-Sn.

Considerando que un vertido con alta DQO potencialmente impactará de manera más severa el cuerpo de agua receptor que uno con alta concentración de color, una propuesta de tratamiento debe valorar entre reducir la coloración o minimizar la DQO. Los autores consideran que la segunda opción debe ser la más recomendable, por lo que se recomienda que el tratamiento se realice con DSA de Ir, cuidando que la corriente aplicada sea suficiente para permitir la generación de los mediadores $\left(\mathrm{HOCl}^{-}, \mathrm{OCl}^{-}\right)$pero que el $\mathrm{pH}$ se mantenga en valores cercanos a la neutralidad. 


\section{Tratamiento de disoluciones de naranja ácido 24 mediante ánodos dimensionalmente estables}

\section{Resumen}

La eliminación del colorante naranja ácido 24 fue estudiada en una celda electroquímica, cuyo ánodo, del tipo dimensionalmente estable (DSA, por sus siglas en inglés), consistió de: a) una malla de titanio cubierta por una capa de iridio (Ir), b) una placa de acero inoxidable recubierta por una capa de una mezcla de iridio-estaño (Ir$\mathrm{Sn})$. El cátodo fue de acero inoxidable y el medio electrolítico fue una disolución conteniendo sulfato sódico, cloruro sódico, así como agentes humectantes y dispersantes requeridos para incorporar el color en la fibra. Los productos comerciales utilizados son: Uniperol y Leophen.

La aproximación experimental permitió visualizar el papel desempeñado por el sulfato, cloruro y surfactantes en la eliminación de color y en la demanda química de oxígeno (DQO). Los resultados obtenidos mostraron que, en medio oxidante de cloruros, la mayor remoción de color, la proveyó el electrodo de Ir-Sn. En el caso de incluir surfactantes, las remociones de color son similares con ambos electrodos. No así el parámetro de DQO, ya que el electrodo de Ir permite obtener una disminución de DQO hasta 6 veces superior a la obtenida con el electrodo de Ir-Sn.

Palabras clave: colorante azo, DSA, agua residual textil, tratamiento electroquímico.

\section{Referencias}

1. R.M. Christie, Color Chemistry, The Royal Society of Chemistry, 2001.

2. United States Environmental Protection Agency (USEPA), Sector notebook proyect: profile of the textile industry, 1997.

3. A. Solano Hernández, Remoción de colorante naranja ácido por el método de elctrocoagulación. Tesis de grado, Facultad de Ingeniería, BUAP, 2005.

4. A. Fernandes, A. Morao, M. Magrinho, A. Lopez, I. Goncalves, Electrochemical degradation of C. I. acid orange 7. Dyes and Pigments 61 (2004) 287-296.

5. I. Szpyrkowicz, Hydrodynamic effects on the performance of the electrocoa-gulation/electroflotation for the removal of dyes from textile wastewater, Ind. Eng. Chem. Res. 44 (2005) 7844-7853.

6. D. Rajkumar, B.J. Song, J.G. Kim, Electrochemical degradation of reactive blue 19 in chloride medium for the treatment of textile dyeing wastewater with identification of intermediate compounds, Dyes and Pigments 72 (2007) 1-7.

7. J. Hastie, J.D. Bejan, M. Teutli, N. Bunce, Electrochemical methods for degradation of Orange II (sodium 4-(2-Hydroxy-1naphtylazo)benzenesulfonate), Ind. Eng. Chem. Res. 45 (8) (2006) 28302839.

8. A. Savall, Chimia 49 (1995) 23-27.

9. Ch. Comninellis, C. Pulgarin, J. Appl. Electrochem. 21 (1991) 703-708.

10. J.D. Rodgers, W. Jedral, N. Bunce, Electrochemical oxidation of chlorinated phenols, Environm. Sci. Technol. 33 (1999) 1453-1457. 
11. S.S. Vaghela, A.D. Jethva, B.B. Mehta, S.P. Dave, S. Adimurthy and G. Ramanchadraiah, Laboratory studies of electrochemical treatment of industrial azo dyes, Environm. Sci. Technol. 39 (2005) 2848-2855.

12. M.J. Kupferle, A. Galai, P.L. Bishop, Electrolytic treatment of azo dye wastewaters: impact of the matrix chloride content, J. Environm. Eng. 132 (5) (2006) 514-518. 